\title{
A Simple Drill for the Pythagorean Theorem with
}

\section{Scissors on Circles!}

\author{
Amitava Biswas \\ SHS School, University of Southern Mississippi, Hattiesburg, MS 39406, USA \\ Abhishek Bisaria \\ Spectral D\&T Inc, Bridgewater, NJ 08807, USA
}

This article is distributed under the Creative Commons by-nc-nd Attribution License.

Copyright (C) 2020 Hikari Ltd.

\begin{abstract}
In this paper the Pythagorean theorem is developed from a constructive exercise with scissors on circles, instead of traditional squares. This approach with circles, naturally involves symmetry and is expected to be easier to remember compared to many traditional proofs.
\end{abstract}

Mathematics Subject Classification: 12D15, 51C05, 51N20, 51M04, $37 \mathrm{E} 10$.

Keywords: Fundamental Geometry; Alternative Perspective; Craft with Mathematics, Classroom Activity, Preliminaries of Calculus

\section{Introduction}

Over time, many mathematicians have presented various thought provoking mathematical perspectives and proofs that may be too broad or general [1-18]. For example, Mnatsakanian [13] presented generalized ideas suitable for arbitrary annular shapes. From these perspectives a non-traditional exercise for the Pythagorean theorem has been developed specifically for circles. As shown in Figure 1, our task is to build a circle of radius $\boldsymbol{A C}$, having the total area of two circles of radii $\boldsymbol{A} \boldsymbol{B}$ and $\boldsymbol{B C}$, which happens to demonstrate the Pythagorean theorem. 


\section{Assumptions}

To handle this problem easily, like most problems in real life, an approximate solution will be done first. Due to its fundamental nature, this exercise minimally assumes that the area of a rectangle is the product of its length and width (see [17]). This exercise also assumes that the ratio between the circumference and the diameter of any circle is always the constant $\mathrm{Pi}$ (see [12]).

\section{The Procedure}

(a) Radially divide the circle of radius $\boldsymbol{A B}$ into many equal sectors, each sector having one sharply pointed tip, and two corners of nearly right angles.

(b) Rearrange the sectors side by side, without gaps or overlaps, perfectly matching each other along the straight edges, but the pointed tip of every sector is oppositely oriented relative to the next sector. When the number of sectors is large, this arrangement looks like a rectangle as a whole, with its width corresponding to the radius and its length corresponding to half of the circumference of the original circle. Therefore, we find that the area of any circle is equal to the square of its radius times the constant $\mathrm{Pi}$. This knowledge will be helpful for understanding following ideas.

(c) Rearrange the sectors side by side, without gaps or overlaps. The pointed tips of all sectors pointing towards the original center. Mutually slide the sectors, touching each other along the straight edges, in a symmetric pattern, creating a central empty polygonal space.

(d) Place the circle of radius $\boldsymbol{B C}$ within that empty polygonal space.

(e) Stop sliding the sectors when the sharply pointed tip of each sector is positioned on the circumference of the circle of radius $\boldsymbol{B C}$.

(f) Obviously, this is an approximate solution to the given problem. The overall shape, including the inner circle of radius $\boldsymbol{B C}$ and all the surrounding sectors, looks like a larger circle, having that total area of the circles of radius $\boldsymbol{A B}$ and $\boldsymbol{B C}$.

(g) An exact solution will be approached in the limit as the number of sectors is increased. That concept happens to be a preliminary step to calculus but not needed further for capturing this simple drill.

(h) Notice in Figure 1, the triangle $\triangle B 1 B 2 C$ is equilateral. The angles $\angle B 1 B 2 C$ and $\angle B 2 B 1 C$ are equal. When the number of sectors is increased these angles will approach the right angle $\llcorner A B C$, giving the right triangle $\triangle \boldsymbol{A B C}$.

(i) We now see that the area of the circle of radius $\boldsymbol{A C}$ is equal to the sum of the 
area of the circle of radius $\boldsymbol{A B}$ and the area of the circle of radius $\boldsymbol{B C}$. Now recall the formula from above step (b). Therefore, the square of $\boldsymbol{A C}$ is equal to the sum of the squares of $\boldsymbol{A B}$ and $\boldsymbol{B C}$. As a result we have an indirect demonstration of the Pythagorean theorem!

\section{Conclusion}

Since many students learn better with visual constructions $[2,4,7,8]$, this graphical representation of the classic theorem could also help instructors enhance interest and understanding of the basic concept among the students and introduce preliminaries of calculus.

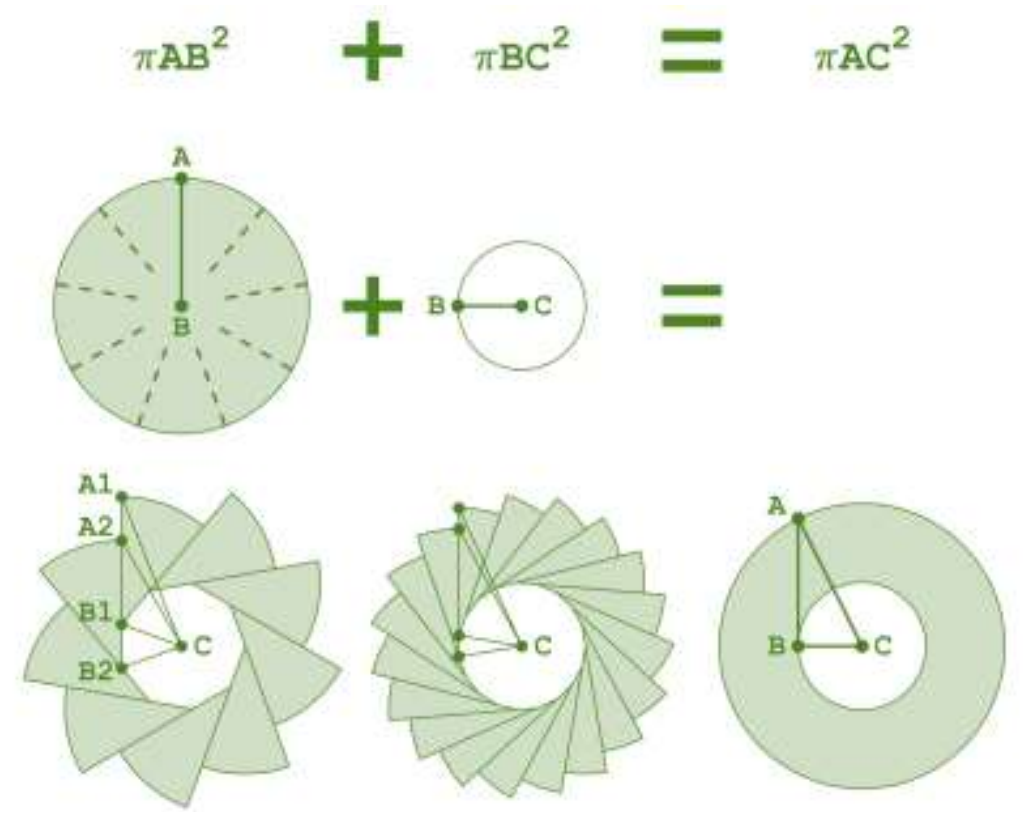

Figure 1. A circle is radially divided into many equal sectors. The sectors are shifted along their straight edges in a symmetric pattern. A right angled triangle $\triangle A B C$ tends to emerge to show the Pythagorean theorem.

Acknowledgements. This work has benefited from faculty start-up funds granted by the University of Southern Mississippi to Dr. Amitava Biswas.

\section{References}

[1] D. Alexander and G. Koeberlein, Elementary Geometry for College Students, 7th Edition. Cengage Learning, Boston, MA, 2019, ISBN-10: 1337614084.

[2] E. Bobek and B. Tversky, Creating visual explanations improves learning, Cognitive Research, 1 (2016). https://doi.org/10.1186/s41235-016-0031-6 
[3] C. Christine, C. Benson, and C. Malm, Bring the Pythagorean Theorem "Full Circle", Mathematics Teaching in the Middle School, 16 (2011), 336-344.

[4] I. Clarke, T. Flaherty, and M. Yankey, Teaching the Visual Learner, Journal of Marketing Education, (2006). https://doi.org/10.1177/0273475306291466

[5] H.Y. Cui, To string together six theorems of physics by Pythagoras theorem, (2002). arXiv:physics/0205021 [physics.gen-ph].

[6] A. Faessler and X. Tu, More around Pythagore from ancient to modern times, International Mathematical Forum, 8 (2013), 1963-1975. https://doi.org/ 10.12988/imf.2013.311217

[7] K. Ha, Getting the Picture: Using Visual Learning Techniques to Foster Higher Order Thinking Skills and Encourage Connections in the Secondary Classroom, Language Arts Journal of Michigan, 10 (2005). https://doi.org/ 10.9707/2168149X.1198

[8] H. Jacobs, Geometry: Seeing, Doing, Understanding, 3rd Edition, Master Books, Green Forest, AR, 2017, ISBN-10: 168344020X.

[9] R. Jurgensen, R. Brown, and J. Jurgensen, Geometry, Houghton Mifflin, Evanston, Illinois, 2000, ISBN-10: 9780395977279.

[10] Z. Lengvárszky, Proving the Pythagorean Theorem via Infinite Dissections, The American Mathematical Monthly, 120 (2013), 751-753. https://doi.org/104169/amer.math.monthly.120.08.751

[11] M.P. Maraner, A Spherical Pythagorean Theorem, The Mathematical Intelligencer, 3 (2010), 46-47. https://doi.org/10.1007/s00283-010-9152-9

[12] MathWikia, Proof: Pi is Constant, https://math.wikia.org/wiki/Proof:_Pi_is_ Constant, Retrieved April 16, 2020.

[13] M. Mnatsakanian, Annular Rings of Equal Area, Math Horizons, 5 (1997), 58. https://doi.org/10.1080/10724117.1997.11975029

[14] L. Okun, The theory of relativity and the Pythagorean theorem, Physics Uspekhi, 51 (2008), 622-623. https://doi.org/10.1070/PU2008v051n06A BEH006552

[15] B, Peterson, Teaching the Pythagorean Theorem for Understanding, The Mathematics Teacher, 103 (2009), 160. ISSN 0025-5769, ISSN-e 2330-0582 
[16] F. Paul, Extending the Pythagorean Theorem to Other Geometries, Mathematics Magazine, 69 (1996), 222-223. https://doi.org/10.1080/ 0025570X.1996. 11996434

[17] ProofWiki, Area of Parallelogram / Rectangle, https://proofwiki.org/wiki/ Area_of_Parallelogram/Rectangle, Retrieved April 16, 2020.

[18] S. Swaminathan, The Pythagorean Theorem, J Biodivers Biopros Dev., 1 (2014), 128-130. https://doi.org/10.4172/2376-0214.1000128

Received: April 5, 2020; Published: April 19, 2020 